\title{
Adsorption of fluoride by porous adsorbents: Estimating pore diffusion coefficients from batch kinetic data
}

\author{
Gang Yuan', Bin Zhao ${ }^{2}$ Khim Hoong $\mathrm{Chu}^{3^{+}}$ \\ ${ }^{1}$ Suzhou Industrial Park Sino French Environmental Technology, Suzhou 215125, People's Republic of China \\ ${ }^{2}$ Dellsen Education \& Technology, Montreal, Quebec, Canada H4C OB8 \\ ${ }^{3}$ Honeychem, Nanjing Chemical Industry Park, Nanjing 210047, People's Republic of China
}

\begin{abstract}
A simple method is presented for extracting pore diffusion coefficients from batch adsorption kinetic data. The method employs the classic Langmuir kinetics model which is characterized by a single rate coefficient. An analytical solution in the form of a simple algebraic equation is available for this rate model. Fitting the algebraic equation to batch kinetic data to determine the rate coefficient is straightforward and can be conveniently accomplished using standard spreadsheet programs. The resultant rate coefficient can be converted to the pertinent pore diffusion coefficient via a separate algebraic expression. The proposed modeling approach provides accurate fits of experimental kinetic data taken from the literature and yields acceptable errors in the best estimates for pore diffusion coefficients. Specific examples discussed are the adsorption of fluoride by bone char and laterite adsorbents.
\end{abstract}

Keywords: Adsorption, Fluoride, Langmuir kinetics, Parameter estimation, Pore diffusion

\section{Introduction}

Adsorption provides an efficient means of removing organic and inorganic contaminants from aqueous solutions [1-6]. The equilibrium and kinetic characteristics of a given adsorption system are usually determined from experimental data obtained from batch contactors. In the case of batch kinetic studies, simple surface reaction models are frequently used by investigators for data analysis. Notable examples include the pseudo first-order or Lagergren kinetic equation and the pseudo second-order kinetic equation. The popularity of these two kinetic equations is due, in part, to the simplicity of their mathematical forms which allow the equations to be linearized for parameter estimation by means of linear regression. The two pseudo reaction rate equations have however encountered criticisms on several fronts. For example, the speciousness of a linearized form of the pseudo second-order equation for parameter estimation has been discussed at some length by a number of investigators [7-9]. Methodological biases and flaws exist in the comparison of the modeling abilities of these two kinetic equations [10-12]. From a theoretical viewpoint, they should be considered as being purely phenomenological with no sound phys- icochemical basis [13]. The physical significance of their rate coefficients has been called into question [14-16]. Also, they are merely curve fitting tools and should not be used to identify the actual uptake mechanism $[17,18]$. Two recent reviews $[19,20]$ as well as a recent textbook on adsorption [21] have summarized the various shortcomings of the two pseudo reaction rate equations.

For porous adsorbent particles the rate of adsorption is often controlled by intraparticle diffusion [22, 23]. Thus, a more instructive approach in the modeling of adsorption kinetics is to use physically reasonable rate models based on the kinetics of mass transfer processes. Rate models accounting for an external film mass transfer resistance and one or more intraparticle diffusion mechanisms allow a realistic mathematical description of the kinetics of many adsorption systems. Because intraparticle diffusion coefficients are an intrinsic property of a given adsorption system, they are independent of the mode of operation. For this reason, diffusion coefficients determined from a batch contactor may be used to model the dynamic behavior of a fixed bed adsorber. In general, two distinct intraparticle transport models, pore diffusion and surface/solid diffusion, are commonly used to describe the kinetics of contaminant adsorption by porous particles. Both dif-
This is an Open Access article distributed under the terms of the Creative Commons Attribution Non-Commercial License (http://creativecommons.org/licenses/by-nc/3.0/) which permits unrestricted non-commercial use, distribution, and reproduction in any medium, provided the original work is properly cited.

Copyright (C) 2020 Korean Society of Environmental Engineers
Received May 13, 2019 Accepted August 31, 2019

${ }^{\dagger}$ Corresponding author

Email: khimchu@gmail.com

Tel: +86-25-58397899 Fax: +86-25-58397899

ORCID: 0000-0003-4012-0667 
fusion models have been used by investigators to fit batch kinetic data with equal accuracy. More sophisticated models with pore and surface diffusion acting in parallel have also been studied by some practitioners [24-26]. From a practical standpoint, it is more convenient to use solid-phase diffusion models based on a single intraparticle diffusion mechanism to model adsorption kinetics because they are computationally simpler.

The present paper deals with an important aspect of pore diffusion: determination of pore diffusion coefficients from experimental data. Batch contactors provide a simple and convenient way to acquire kinetic data. By fitting a pore diffusion model to such data, the pore diffusion coefficient of the model can be determined. However, for nonlinear systems the data fitting process is often complicated by the need to solve the model equations numerically $[27,28]$. As an alternative, the present article explores the feasibility of using a simple kinetic model to extract pore diffusivities from batch kinetic data without resorting to numerical methods. The approximation is based on the use of the classic Langmuir kinetics model - which reduces to the Langmuir isotherm model at large values of time - to describe the rate of uptake. Although the Langmuir kinetics model is based on a surface reaction mechanism, its rate coefficient, unlike those of the pseudo first-order and pseudo second-order kinetic equations, can be converted to the pertinent pore diffusion coefficient [29-31].

For batch adsorption systems, it is possible to derive from the Langmuir kinetics model an analytical expression in the form of an algebraic equation. Fitting the algebraic equation to batch kinetic data yields the rate coefficient of the Langmuir kinetics model, from which the corresponding pore diffusion coefficient may be computed. The data fitting process can be easily accomplished using standard spreadsheet programs. Using data taken from the environmental adsorption literature, the potential of this simple modeling approach was evaluated here on its ability to provide accurate pore diffusion coefficients of fluoride ions. Elevated levels of fluoride in drinking water are a major public health concern in many parts of the world. Adsorption has been recognized as an effective technique for the removal of fluoride from water sources [32-34].

\section{Theory}

\subsection{Film-Pore Diffusion Model}

In this model the transport of a solute within a spherical particle is described by pore diffusion and is given by the following equation and initial and boundary conditions [22, 23]:

$$
\begin{gathered}
\varepsilon_{p} \frac{\partial c}{\partial t}+\rho_{p} \frac{\partial q}{\partial t}=\frac{D_{p}}{r^{2}} \frac{\partial}{\partial r}\left(r^{2} \frac{\partial c}{\partial r}\right) \\
t=0 \quad c=0 \quad q=0 \\
r=0 \quad \frac{\partial c}{\partial r}=0 \\
r=R_{p} \quad D_{p} \frac{\partial c}{\partial r}=k_{f}(C-c)
\end{gathered}
$$

In these equations, $C$ and $C$ are respectively the pore fluid and bulk solution concentrations at time $t, q$ is the adsorbed phase concentration at time $t, \varepsilon_{p}$ is the particle porosity, $\rho_{p}$ is the particle density, $D_{p}$ is the pore diffusion coefficient, $k_{f}$ is the external mass transfer coefficient, $r$ is the particle radial coordinate, and $R_{p}$ is the particle radius. Eq. (1(c)) describes mass transfer through a boundary film surrounding an individual particle.

The conservation equation and initial condition for a batch contactor are given as [22, 23]

$$
\begin{gathered}
\frac{\mathrm{d} C}{\mathrm{~d} t}=-\frac{3 k_{f}}{R_{p}} \frac{m}{V}\left(C-\left.c\right|_{r=R_{p}}\right)=-\frac{m}{V} \frac{\mathrm{d} \bar{q}}{\mathrm{~d} t} \\
t=0 \quad C=C_{o}
\end{gathered}
$$

where $m$ is the mass of adsorbent, $V$ is the volume of solution, $\bar{q}$ is the solute concentration in the adsorbent particle averaged over the particle volume, and $C_{o}$ is the initial concentration. The relationship between adsorbed phase and pore fluid concentrations is defined by the following Langmuir isotherm model [35]:

$$
q=\frac{q_{m} b c}{1+b c}
$$

where $q_{m}$ and $b$ are respectively the Langmuir saturation capacity and Langmuir constant. These equations are nonlinear and must, in general, be solved numerically.

\subsection{Langmuir Kinetics Modeling Approach}

According to the classic Langmuir kinetics model [36, 37], the interaction between a solute and an adsorption site is described by the following reaction rate equation:

$$
\frac{\mathrm{d} \bar{q}}{\mathrm{~d} t}=k_{1} C\left(q_{m}-\bar{q}\right)-k_{2} \bar{q}
$$

where $k_{1}$ is a second-order forward rate coefficient and $k_{2}$ is a first-order reverse rate coefficient. At equilibrium the derivative $d \bar{q} / d t$ approaches zero and Eq. (4) reduces to the Langmuir isotherm model given by Eq. (3) with $b=k_{1} / k_{2}$. Eq. (4) may be rewritten as

$$
\frac{\mathrm{d} \bar{q}}{\mathrm{~d} t}=k_{1}\left[C\left(q_{m}-\bar{q}\right)-\frac{\bar{q}}{b}\right]
$$

Integration of Eqs. (2) and (5) yields the following analytical solution [38]:

$\frac{C}{C_{o}}=1-\frac{(m / V)(\alpha-\beta)}{C_{o}}\left\{\frac{1-\exp \left[-(2 \beta m / V) k_{1} t\right]}{1-[(\alpha-\beta) /(\alpha+\beta)] \exp \left[-(2 \beta m / V) k_{1} t\right]}\right\}$

where

$$
\alpha=\frac{1}{2}\left(C_{o} \frac{V}{m}+q_{m}+\frac{1}{b} \frac{V}{m}\right) \quad \beta=\sqrt{\alpha^{2}-C_{o} q_{m} \frac{V}{m}}
$$


For an experimental kinetic test, the parameters $C_{o}, m$, and $V$ are known while the parameters $\alpha$ and $\beta$ may be computed with known Langmuir isotherm parameters $q_{m}$ and $b$, and so the rate coefficient $k_{1}$ is the only fitting parameter which may be obtained by matching Eq. (6) to the experiment's data. Note that Eq. (6) may be rearranged in the following manner to allow estimation of $k_{1}$ by means of linear regression:

$$
\frac{1}{2} \frac{1}{\beta} \frac{V}{m} \ln \left[\frac{\left(C_{o}-C\right) /(\alpha+\beta)-m / V}{\left(C_{o}-C\right) /(\alpha-\beta)-m / V}\right]=k_{1} t
$$

A plot of the left-hand side of Eq. (7) versus $t$ should be linear with slope $k_{1}$ [39].

In adsorption studies $k_{1}$ is often regarded as a "lumped" kinetic rate constant which includes all mass transfer resistances (film diffusion, surface diffusion, pore diffusion, and surface reaction resistance). Here, we restrict our interest to the film mass transfer and pore diffusion resistances which can be combined in the following manner [29-31]:

$$
\frac{1}{k_{1}}=\frac{R_{p}^{2} \rho_{p} q_{m}(R+1)}{30}\left(\frac{5}{k_{f} R_{p}}+\frac{1}{D_{p}}\right)
$$

where,

$$
R=\frac{1}{1+b C_{o}}
$$

Given $k_{1}$, the film mass transfer coefficient $k_{f}$, and other relevant parameters, the pore diffusion coefficient $D_{p}$ may be calculated from Eq. (8). An expression similar to Eq. (8) for $k_{1}$ which combines the film mass transfer and surface diffusion resistances are available in the literature [40].

\subsection{Goodness-of-Fit Measure}

The following coefficient of determination (COD) gives a good idea of the overall goodness-of-fit of the Langmuir kinetics model to measured data:

$$
\mathrm{COD}=\frac{\sum_{j=1}^{w}\left(z_{j}^{\text {mod }}-\bar{z}^{\text {exp }}\right)^{2}}{\sum_{j=1}^{w}\left(z_{j}^{m o d}-\bar{z}^{e x p}\right)^{2}+\sum_{j=1}^{w}\left(z_{j}^{m o d}-z_{j}^{e x p}\right)^{2}}
$$

where $z_{j}^{\text {mod }}$ is the model prediction for observation $j, z_{j}^{e x p}$ is the experimental data for observation $j$, and $\bar{z}^{\exp }$ is the mean of experimental data. A COD score of unity indicates a perfect fit.

\section{Results and Discussion}

Intraparticle diffusion coefficients (pore and surface) serve as important input parameters to solid-phase mass transfer models which are often used in fixed bed adsorber design and analysis. In addition, these diffusion coefficients may be used to compare the kinetic properties of different adsorbents. For a given contaminant removal process, it is important to select an adsorbent which exhibits not only optimal adsorption capacity but also fast uptake kinetics. As such, the subject matter of this work is of considerable practical interest.

Three literature batch data sets obtained for the uptake of fluoride by porous adsorbents have been used in this work to evaluate the performance of the proposed Langmuir kinetics modeling approach. In the selected literature reports the pore diffusion coefficient for each data set has been obtained by fitting either a numerical film-pore diffusion model or a numerical shrinking core model to the kinetic data.

An attractive feature of the Langmuir kinetics model is its ability to account for the effects of isotherm nonlinearity. As noted previously, at equilibrium the Langmuir kinetics model reduces to the Langmuir isotherm model, so the effects of isotherm nonlinearity are inherently included within the rate model. Since many experimental adsorption isotherms follow the Langmuir isotherm model, the Langmuir kinetics model can thus be used to describe the kinetics as well as the equilibrium characteristics of these systems. For rate models with a nonlinear isotherm, a numerical solution is in general required. The Langmuir kinetics model is an exception in this respect since it can be solved analytically. However, the Langmuir kinetics model cannot extract pore diffusion coefficients directly from experimental data; they are instead computed from Eq. (8).

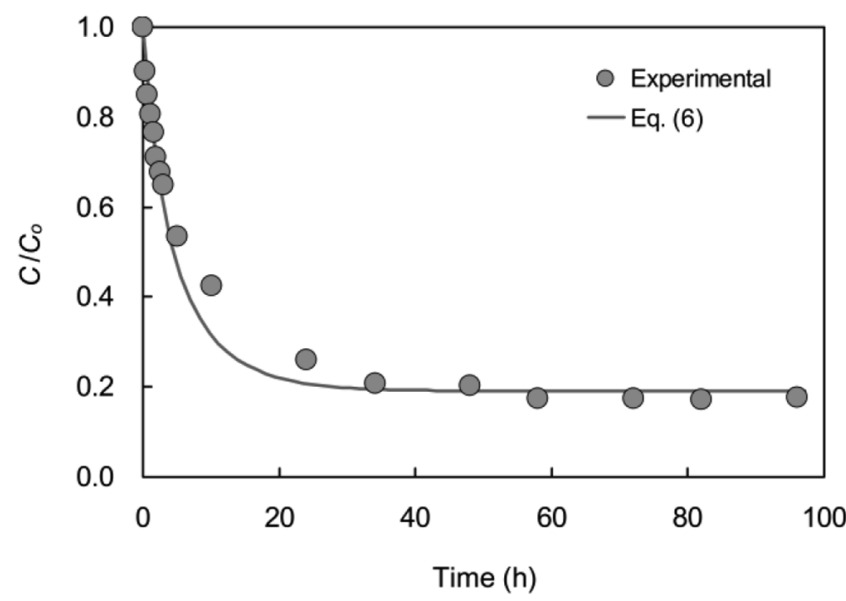

Fig. 1. Adsorption of fluoride by bone char showing comparison between the kinetic data of Nigri et al. [41] and theoretical curve computed from Eq. (6). $\mathrm{COD}=0.986$.

\subsection{Case 1: Adsorption of Fluoride by Bone Char}

Nigri et al. [41] measured the uptake of fluoride by a commercial bone char adsorbent as a function of time using Erlenmeyer flasks containing fluoride solutions and bone char which were placed on a thermostatic orbital shaker. The resultant kinetic data set is shown in Fig. 1. The authors extracted $D_{p}$ from the Fig. 1 data by means of a pore diffusion model which was solved numerically. The numerical solution is based on the explicit finite difference 
method of lines. The optimal value of $D_{p}$ so obtained is 7.15 $\times 10^{-6} \mathrm{~cm}^{2} / \mathrm{s}$. In their modeling approach, Nigri et al. [41] assumed negligible resistance to external film mass transfer. As a result, the pore diffusion model defined by Eq. (1) reduces to their pore diffusion model by omitting the external mass transfer mechanism.

In the Langmuir kinetics modeling approach, determination of $D_{p}$ is based on two distinct steps: estimation of $k_{1}$ by fitting Eq. (6) to batch kinetic data followed by computation of $D_{p}$ from Eq. (8) using the resultant $k_{1}$ value. To extract $k_{1}$ from the Fig. 1 data via Eq. (6), one requires values of the Langmuir isotherm parameters $q_{m}$ and $b$ and two system parameters $C_{o}$ and $m / V$. These parameter values, given in the paper by Nigri et al. [41], are $q_{m}$ $=6.7 \mathrm{mg} / \mathrm{g}, b=800 \mathrm{~cm}^{3} / \mathrm{g}, C_{o}=1 \times 10^{-2} \mathrm{~g} / \mathrm{cm}^{3}$, and $\mathrm{m} / V=$ $2 \times 10^{-3} \mathrm{~g} / \mathrm{cm}^{3}$.

Since the Langmuir isotherm parameters serve as input parameters to Eq. (6), it is important to check whether the Fig. 1 kinetic data are consistent with prediction of the Langmuir isotherm model. This consistency will ensure a good fit of Eq. (6) to the data points of Fig. 1 collected at large values of time when apparent equilibrium was established. The equilibrium concentration at large values of time, $C_{e}$, for a batch adsorber that follows the Langmuir isotherm may be computed from the following expression:

$$
C_{e}=\frac{\sqrt{h^{2}+\frac{4 C_{o}}{b}}-h}{2}
$$

where

$$
h=\frac{1}{b}+\frac{m}{V} q_{m}-C_{o}
$$

The value of $C_{e}$ for the batch kinetic experiment of Nigri et al. [41] computed from Eq. (10) is $1.9 \times 10^{-3} \mathrm{mg} / \mathrm{cm}^{3}$, which agrees with the observed $C_{e}$ value of approximately $1.8 \times 10^{-3} \mathrm{mg} / \mathrm{cm}^{3}$ (indicated by the last data point of Fig. 1). Thus, it is evident that the Langmuir parameters $q_{m}$ and $b$ extracted from independent equilibrium experiments are able to describe the equilibrium state of the batch kinetic experiment with good accuracy.

Eq. (6) was fitted to the Fig. 1 data by a nonlinear regression procedure using the $q_{m}, b, C_{o}$, and $m / V$ values given above, yielding $k_{1}=4.38 \times 10^{-3} \mathrm{~cm}^{3} / \mathrm{mg} \mathrm{s}$ and a COD score of 0.986 . As seen in Fig. 1, the entire data set is fitted well by Eq. (6). Having determined $k_{1}$, it is straightforward to compute $D_{p}$ from Eq. (8), which has several other input parameters. Values of $q_{m}, b$, and $C_{o}$ are listed above while values of the adsorbent radius $R_{p}$ and adsorbent density $\rho_{p}$, as reported by Nigri et al. [41], are respectively $0.07 \mathrm{~cm}$ (mean) and $0.96 \mathrm{~g} / \mathrm{cm}^{3}$. The remaining parameter of Eq. (8), the external mass transfer coefficient $k_{f}$, is not available since Nigri et al. [41] assumed negligible external mass transfer in their modeling approach.

Because the initial uptake of solute is controlled only by the external mass transfer resistance, $k_{f}$ may be estimated from experimental values of $C / C_{o}$ obtained during the initial uptake period. The fluid phase concentration change during the initial period is given by [42, 43]:

$$
\frac{C}{C_{o}}=1-\frac{3 k_{f} m}{R_{p} \rho_{p} V} t
$$

The preceding equation implies that a plot of $C / C_{o}$ against $t$ for small times gives a straight line with slope $\left(3 m / R_{p} \rho_{p} V\right) k_{f}$. Therefore, $k_{f}$ can be calculated from such a slope. We calculated $k_{f}$ as $1.01 \times 10^{-3} \mathrm{~cm} / \mathrm{s}$ by fitting Eq. (11) to the first two data points of Fig. 1.

Knowing all the relevant parameters, the pore diffusion coefficient can be computed from Eq. (8): $D_{p}=8.03 \times 10^{-6} \mathrm{~cm}^{2} / \mathrm{s}$. This $D_{p}$ value obtained by the Langmuir kinetics modeling approach and the $D_{p}$ value determined by Nigri et al. [41] differ only by $12 \%$, as shown in Table 1 . The good agreement confirms the validity of the proposed modeling approach.

Table 1. Comparison between $D_{p}$ Values Obtained by the Langmuir Kinetics Modeling Approach and Literature $D_{p}$ Values

\begin{tabular}{lccc}
\hline \multirow{2}{*}{ Adsorption system } & \multicolumn{2}{c}{$D_{\boldsymbol{p}}\left(\mathbf{c m}^{2} / \mathbf{s}\right)$} & \multirow{2}{*}{ Error (\%) } \\
\cline { 2 - 4 } & Literature & This work & \\
\hline Fluoride-bone char [41] & $7.15 \times 10^{-6}$ & $8.03 \times 10^{-6}$ & 12 \\
Fluoride-bone char [44] & $3.31 \times 10^{-6}$ & $3.04 \times 10^{-6}$ & 8 \\
Fluoride-laterite [45] & $2.3 \times 10^{-6}$ & $1.81 \times 10^{-6}$ & 21 \\
\hline
\end{tabular}

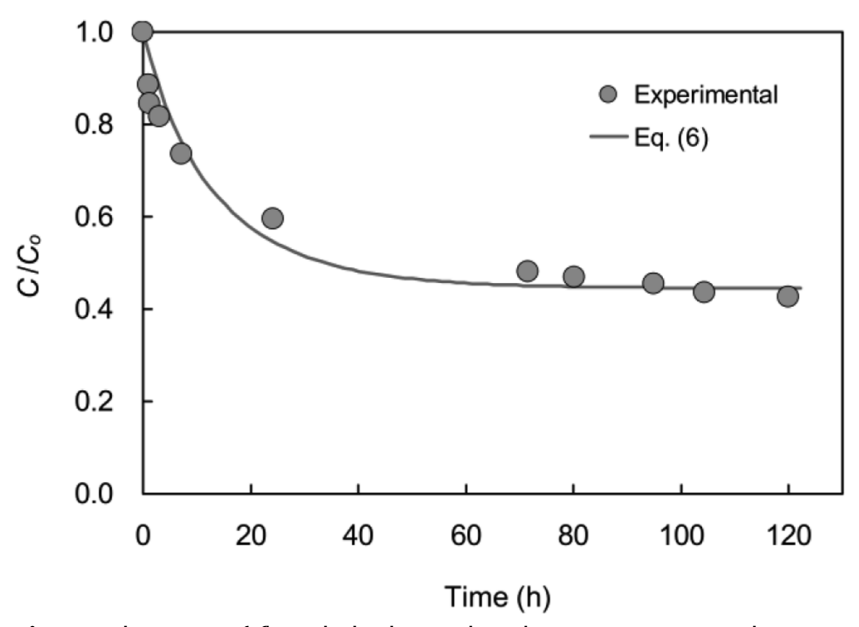

Fig. 2. Adsorption of fluoride by bone char showing comparison between the kinetic data of Leyva-Ramos et al. [44] and theoretical curve computed from Eq. (6). COD $=0.961$.

\subsection{Case 2: Adsorption of Fluoride by Bone Char}

The uptake of fluoride by a commercial bone char adsorbent has also been investigated by Leyva-Ramos et al. [44] who employed a rotating basket batch adsorber to obtain kinetic data for a series of experimental runs with varying system and operational conditions. We will focus on a particular kinetic experiment (run 13 in Fig. 6 of their paper). The adsorbent size of this run is similar to that of case 1. The authors fitted the film-pore diffusion model given by Eq. (1) to the chosen kinetic data set, shown in Fig. 2, obtaining $D_{p}=3.31 \times 10^{-6} \mathrm{~cm}^{2} / \mathrm{s}$. The partial and ordinary differential equations of the film-pore diffusion model (Eqs. (1) and (2)) 
were numerically solved by using a specialized software package. The external mass transfer coefficient $k_{f}$ - required by the fitting procedure as one of the model inputs - was estimated from data points in the small time region which yielded $k_{f}=3.07 \times 10^{-3}$ $\mathrm{cm} / \mathrm{s}$. Other relevant system and operational parameters are as follows: $C_{o}=5.66 \times 10^{-3} \mathrm{~g} / \mathrm{cm}^{3}, \mathrm{~m} / V=1 \times 10^{-3} \mathrm{~g} / \mathrm{cm}^{3}, R_{p}=$ $0.065 \mathrm{~cm}$, and $\rho_{p}=0.96 \mathrm{~g} / \mathrm{cm}^{3}$. Note that the $\rho_{p}$ value is taken from case 1 since it is not given in the paper by Leyva-Ramos et al. [44].

As in case 1, one should verify the ability of the Langmuir isotherm to predict the apparent equilibrium state of the kinetic data set of Fig. 2 before fitting Eq. (6) to the kinetic data. The final data point of Fig. 2 gives an experimental $C_{e}$ value of approximately $2.4 \times 10^{-3} \mathrm{mg} / \mathrm{cm}^{3}$. According to Leyva-Ramos et al. [44], the Langmuir isotherm parameters $q_{m}$ and $b$ extracted from batch equilibrium data are $5.89 \mathrm{mg} / \mathrm{g}$ and $450 \mathrm{~cm}^{3} / \mathrm{g}$, respectively. The theoretical $C_{e}$ value calculated from Eq. (10) using these two isotherm parameters is $2.5 \times 10^{-3} \mathrm{mg} / \mathrm{cm}^{3}$, confirming the validity of the Langmuir isotherm parameters. Knowing $C_{o}, m / V, q_{m}$, and $b$, the unknown rate coefficient $k_{1}$ can be obtained by fitting Eq. (6) to the Fig. 2 data. The best-fit value of $k_{1}$ is $2.19 \times 10^{-3} \mathrm{~cm}^{3} / \mathrm{mg}$ s. A comparison between experimental and calculated curves is shown in Fig. 2 from which it may be seen that the Langmuir kinetics model provides a satisfactory fit of the experimental data $(\mathrm{COD}=0.961)$

We calculated $k_{f}$ as $6.17 \times 10^{-4} \mathrm{~cm} / \mathrm{s}$ by fitting Eq. (11) to the first two data points of Fig. 2. Note that our $k_{f}$ value is noticeably smaller than the $k_{f}$ value obtained by Leyva-Ramos et al. [44]. The difference may be due to the use of different initial data points to extract $k_{f}$ from the small time region. We were not able to extract accurate initial data points from the original figure because it contains several cluttered data points in the small time region. Also, as noted above we have used an assumed value for the adsorbent density $\rho_{p}$ in the calculation of $k_{f}$ from Eq. (11). Knowing $k_{1}, k_{f}$, $q_{m}, b, C_{o}, R_{p}$, and $\rho_{p}$, the pore diffusion coefficient $D_{p}$ can be computed from Eq. (8) as $3.04 \times 10^{-6} \mathrm{~cm}^{2} / \mathrm{s}$. The $D_{p}$ value obtained here is in excellent agreement with that determined by Leyva-Ramos et al. [44], showing a deviation of $8 \%$ (see Table 1).

It is interesting to note that the literature $D_{p}$ value for case $1\left(7.15 \times 10^{-6} \mathrm{~cm}^{2} / \mathrm{s}\right)$ is about two times larger than the literature $D_{p}$ value for case $2\left(3.31 \times 10^{-6} \mathrm{~cm}^{2} / \mathrm{s}\right)$. This difference is somewhat unexpected given that the textural properties of the two commercial bone char adsorbents are quite similar, as shown in Table 2. In principle, adsorbents with similar textural properties should exhibit similar pore diffusivities.

Table 2. Textural Properties of Two Commercial Bone Char Adsorbents

\begin{tabular}{lcc}
\hline Reference & Nigri et al. [41] & Leyva-Ramos et al. [44] \\
\hline Raw material & Cow bone & Cow bone \\
\hline Manufacturer & BONECHAR, Brazil & APELSA, Mexico \\
\hline Particle size $(\mathrm{mm})$ & $1.1-1.7($ mean $=1.4)$ & 1.29 \\
\hline Surface area $\left(\mathrm{m}^{2} / \mathrm{g}\right)$ & 138.8 & 104 \\
\hline Particle density $\left(\mathrm{g} / \mathrm{cm}^{3}\right)$ & 0.96 & - \\
\hline Total pore volume $\left(\mathrm{cm}^{3} / \mathrm{g}\right)$ & 0.308 & 0.30 \\
\hline Average pore diameter $(\mathrm{nm})$ & 8.86 & 11.1 \\
\hline
\end{tabular}

\subsection{Case 3: Adsorption of Fluoride by Laterite}

Batch experiments were conducted by Maiti et al. [45] to obtain kinetic data of fluoride adsorption in a chemically treated laterite adsorbent. Three kinetic data sets obtained at different temperatures are available in their paper. The kinetic data set measured at $15{ }^{\circ} \mathrm{C}$ is presented here for analysis (Fig. 3). The experimental conditions are $C_{o}=1 \times 10^{-2} \mathrm{~g} / \mathrm{cm}^{3}$ and $\mathrm{m} / V=5 \times 10^{-4} \mathrm{~g} / \mathrm{cm}^{3}$. The properties of the adsorbent are $R_{p}=0.01 \mathrm{~cm}$ and $\rho_{p}=1.08 \mathrm{~g} / \mathrm{cm}^{3}$. The authors adopted a shrinking core model to describe pore diffusion and external mass transfer. The advantage of this model is that it can be formulated in terms of ordinary differential equations, avoiding the need to solve the partial differential equation given by Eq. (1). The shrinking core model was numerically solved and fitted to the kinetic data of Fig. 3, yielding $D_{p}=2.3 \times 10^{-6} \mathrm{~cm}^{2} / \mathrm{s}$ and $k_{f}=8.3 \times 10^{-2} \mathrm{~cm} / \mathrm{s}$.

By fitting Eq. (3) to independent equilibrium data, Maiti et al. [45] obtained $q_{m}=36.31 \mathrm{mg} / \mathrm{g}$ and $b=230 \mathrm{~cm}^{3} / \mathrm{mg}$. This maximum adsorption capacity is about five to six times larger than the $q_{m}$ values of the two bone char adsorbents analyzed in the previous two cases. Using known values of $C_{o}, m / V, q_{m}$, and $b$, Eq. (6) was fitted to the Fig. 3 data to determine $k_{1}$. The resultant $k_{1}$ is 6.54 $\times 10^{-3} \mathrm{~cm}^{3} / \mathrm{mg} \mathrm{s}$. As seen in Fig. 3, the calculated curve of the Langmuir kinetics model with a COD score of 0.957 is capable of fitting the data reasonably well. The external mass transfer coefficient $k_{f}$ was again determined by fitting Eq. (11) to the first two data points of Fig. 3. Knowing $k_{1}, k_{f}, q_{m}, b, C_{o}, R_{p}$, and $\rho_{p}$, the pore diffusion coefficient $D_{p}$ can be calculated from Eq. (8) as $1.81 \times 10^{-6} \mathrm{~cm}^{2} / \mathrm{s}$. The $D_{p}$ value obtained by our approach is consistent with that obtained by Maiti et al. [45]; they differ by $21 \%$ (see Table 1). It should be noted that the shrinking core model used by Maiti et al. [45] to estimate their $D_{p}$ value is based on a number of assumptions and simplifications. As a result, their $D_{p}$ value could differ from the $D_{p}$ value of the formal film-pore diffusion model given by Eq. (1), to which our $D_{p}$ should be compared.

Fig. 3 reveals that the fluoride-laterite adsorption system needed about $10 \mathrm{~h}$ to reach apparent equilibrium. By contrast, the contact time needed by the two fluoride-bone char adsorption systems

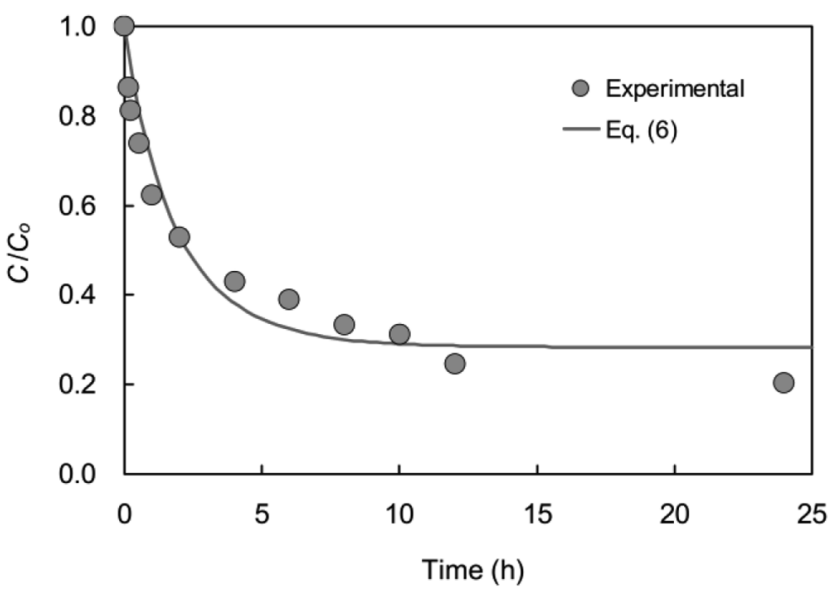

Fig. 3. Adsorption of fluoride by laterite showing comparison between the kinetic data of Maiti et al. [45] and theoretical curve computed from Eq. (6). $\mathrm{COD}=0.957$. 
to reach apparent equilibrium was more than $50 \mathrm{~h}$, as may be seen in Figs. 1 and 2. This marked difference is due to the fact that the particle size of the laterite adsorbent $(0.2 \mathrm{~mm})$ is very much smaller than those of the bone char adsorbents (Table 2). This particle size effect implies that the intraparticle diffusion mechanism played a dominant role in the overall adsorption rates of fluoride in these porous adsorbents.

\subsection{Errors in Fitted $D p$ Values}

On the whole the parameter estimation method presented in this work has proven to be satisfactory, providing a simple and convenient way to extract $D_{p}$ from batch kinetic data without resorting to numerical methods. The fitted $D_{p}$ values differ from literature values by $8-21 \%$ (Table 1). These deviations are in line with errors reported in other similar studies. Differences in the range 3-23\% have been reported by Chu [40] who used the Langmuir kinetics modeling approach to extract surface diffusion coefficients from batch kinetic data. Yao and Chen [46] developed a simplified method based on algebraic equations for estimating surface diffusion coefficients from batch kinetic data and reported errors in the range $11-24 \%$. For the uptake of fluoride by bone char in batch adsorbers, Leyva-Ramos et al. [44] demonstrated that concentration decay curves predicted with best-fit $D_{p}$ values are very similar to those predicted with non-optimal $D_{p}$ values that differ from the best-fit values by $10-17 \%$ (see Fig. 4 of their paper). The magnitude of the errors in $D_{p}$ reported here is therefore deemed tolerable.

\section{Conclusions}

From our evaluation of the Langmuir kinetics modeling approach considered here, we conclude that it affords a simple and convenient way to extract pore diffusion coefficients from batch kinetic data and, with limited computational effort, gives acceptable quantitative results. For the three case studies dealing with the uptake of fluoride by bone char and laterite adsorbents, the fitted pore diffusion coefficients are in good agreement with literature results. The Langmuir kinetics modeling approach requires accurate Langmuir isotherm parameters as well as external mass transfer coefficients which serve as important input parameters. The major drawback of this simple method is that it is restricted to adsorption systems in which the adsorption equilibrium behavior follows the Langmuir isotherm. Additional studies using adsorption systems dealing with other water contaminants such as heavy metals and dyes will be required to expand the evaluation presented here and to confirm the utility of the simple method as a rapid and reliable modeling tool for estimating pore diffusion coefficients from batch kinetic data.

\section{Nomenclature}

$b \quad$ Langmuir constant $\left(\mathrm{cm}^{3} / \mathrm{mg}\right)$

C Solute concentration in pore fluid $\left(\mathrm{mg} / \mathrm{cm}^{3}\right)$

$C \quad$ Solute concentration in bulk fluid $\left(\mathrm{mg} / \mathrm{cm}^{3}\right)$

$C_{e} \quad$ Equilibrium concentration $\left(\mathrm{mg} / \mathrm{cm}^{3}\right)$
$C_{o} \quad$ Initial value of $C\left(\mathrm{mg} / \mathrm{cm}^{3}\right)$

$D_{p} \quad$ Pore diffusion coefficient $\left(\mathrm{cm}^{2} / \mathrm{s}\right)$

$h \quad$ Parameter defined by Eq. (10(a))

$k_{1} \quad$ Langmuir second order forward rate coefficient $\left(\mathrm{cm}^{3} / \mathrm{mg} \mathrm{s}\right)$

$k_{2} \quad$ Langmuir first order reverse rate coefficient (1/s)

$k_{f} \quad$ External film mass transfer coefficient $(\mathrm{cm} / \mathrm{s})$

$m$ Mass of particles (g)

$q \quad$ Solute concentration in particle (mg/g)

$q_{m} \quad$ Langmuir saturation capacity (mg/g)

$\bar{q} \quad$ Particle-average solute concentration (mg/g)

$r \quad$ Particle radial coordinate $(\mathrm{cm})$

$R \quad$ Separation factor defined by Eq. (8(a))

$R_{p} \quad$ Particle radius (cm)

$t \quad$ Time (s)

$V \quad$ Bulk solution volume $\left(\mathrm{cm}^{3}\right)$

$z_{j}^{e x p}$ Experimental data $\left(C / C_{o}\right)$ for observation $j$

$z_{j}^{\text {mod }} \quad$ Model prediction $\left(C / C_{o}\right)$ for observation $j$

$\bar{z}^{\exp }$ Mean of experimental data $\left(C / C_{o}\right)$

\section{Greek Letters}

$a \quad$ Parameter defined by Eq. (6(a)) (mg/g)

$\beta$ Parameter defined by Eq. (6(a)) (mg/g)

$\varepsilon_{p} \quad$ Porosity of particle

$\rho_{p} \quad$ Density of particle $\left(\mathrm{g} / \mathrm{cm}^{3}\right)$

\section{References}

1. Vera LM, Bermejo D, Uguña MF, Garcia N, Flores M, González E. Fixed bed column modeling of lead(II) and cadmium(II) ions biosorption on sugarcane bagasse. Environ. Eng. Res. 2019;24: 31-37.

2. Choi H-J, Yu S-W. Biosorption of methylene blue from aqueous solution by agricultural bioadsorbent corncob. Environ. Eng. Res. 2019;24:99-106.

3. Wi H, Kang S-W, Hwang Y. Immobilization of Prussian blue nanoparticles in acrylic acid-surface functionalized poly(vinyl alcohol) sponges for cesium adsorption. Environ. Eng. Res. 2019;24:173-179.

4. Choi YK, Jang HM, Kan E, Wallace AR, Sun W. Adsorption of phosphate in water on a novel calcium hydroxide-coated dairy manure-derived biochar. Environ. Eng. Res. 2019;24:434-442.

5. Jang HM, Yoo S, Park S, Kan E. Engineered biochar from pine wood: Characterization and potential application for removal of sulfamethoxazole in water. Environ. Eng. Res. 2019;24: 608-617.

6. Singh S, Srivastava VC, Goyal A, Mall ID. Breakthrough modeling of furfural sorption behavior in a bagasse fly ash packed bed. Environ. Eng. Res. 2020;25:104-113.

7. McCuen RH, Surbeck CQ. An alternative to specious linearization of environmental models. Water Res. 2008;42:4033-4040.

8. Zhang J-Z. Avoiding spurious correlation in analysis of chemical kinetic data. Chem. Commun. 2011;47:6861-6863.

9. Xiao Y, Azaiez J, Hill JM. Erroneous application of pseudo-sec- 
ond-order adsorption kinetics model: Ignored assumptions and spurious correlations. Ind. Eng. Chem. Res. 2018;57:2705-2709.

10. Pan B, Xing B. Adsorption kinetics of $17 \alpha$-ethinyl estradiol and bisphenol A on carbon nanomaterials. I. Several concerns regarding pseudo-first order and pseudo-second order models. J. Soil. Sediments 2010;10:838-844.

11. Canzano S, Iovino P, Leone V, Salvestrini S, Capasso S. Use and misuse of sorption kinetic data: A common mistake that should be avoided. Adsorpt. Sci. Technol. 2012;30:217-225.

12. Simonin JP. On the comparison of pseudo-first order and pseudo-second order rate laws in the modeling of adsorption kinetics. Chem. Eng. J. 2016;300: 254-263.

13. Douven S, Paez CA, Gommes CJ. The range of validity of sorption kinetic models. J. Colloid Interf. Sci. 2015;448:437-450.

14. Rodrigues AE, Silva CM. What's wrong with Lagergreen pseudo first order model for adsorption kinetics? Chem. Eng. J. 2016;306:1138-1142.

15. Tien C, Ramarao BV. On the significance and utility of the Lagergren model and the pseudo second order model of batch adsorption. Sep. Sci. Technol. 2017;52:975-986.

16. Tien C, Ramarao BV. Further examination of the relationship between the Langmuir kinetics and the Lagergren and the second-order rate models of batch adsorption. Sep. Purif. Technol. 2014;136:303-308

17. Inglezakis VJ, Fyrillas MM, Park J. Variable diffusivity homogeneous surface diffusion model and analysis of merits and fallacies of simplified adsorption kinetics equations. J. Hazard. Mater. 2019;367:224-245.

18. Wang Z, Giammar DE. Tackling deficiencies in the presentation and interpretation of adsorption results for new materials. Environ. Sci. Technol. 2019;53:5543-5544.

19. Tan KL, Hameed BH. Insight into the adsorption kinetics models for the removal of contaminants from aqueous solutions. $J$. Taiwan Inst. Chem. Eng. 2017;74:25-48.

20. Tran HN, You S-J, Hosseini-Bandegharaei A, Chao H-P. Mistakes and inconsistencies regarding adsorption of contaminants from aqueous solutions: A critical review. Water Res. 2017;120:88-116.

21. Tien C. Introduction to adsorption: Basics, analysis, and applications. Amsterdam: Elsevier; 2019. p. 147-152.

22. Ruthven DM. Principles of adsorption and adsorption processes. New York: Wiley; 1984. p. 166-205.

23. Suzuki M. Adsorption engineering. Tokyo: Kodansha; 1990. p. 95-124.

24. Kavand M, Asasian N, Soleimani M, Kaghazchi T, Bardestani R. Film-pore-[concentration-dependent] surface diffusion model for heavy metal ions adsorption: Single and multi-component systems. Process Saf. Environ. Prot. 2017;107:486-497.

25. Ocampo-Perez R, Aguilar-Madera CG, Díaz-Blancas V. 3D modeling of overall adsorption rate of acetaminophen on activated carbon pellets. Chem. Eng. J. 2017;321:510-520.

26. Souza PR, Dotto GL, Salau NPG. Detailed numerical solution of pore volume and surface diffusion model in adsorption systems. Chem. Eng. Res. Des. 2017;122:298-307.

27. Costa C, Rodrigues A. Intraparticle diffusion of phenol in macro- reticular adsorbents: Modeling and experimental study of batch and CSTR adsorbers. Chem. Eng. Sci. 1985;40:983-993.

28. Leyva-Ramos R, Geankoplis CJ. Diffusion in liquid-filled pores of activated carbon. I. Pore volume diffusion. Can. J. Chem. Eng. 1994:37:262-271.

29. Hiester NK, Vermeulen T. Saturation performance of ion exchange and adsorption columns. Chem. Eng. Prog. 1952;48:505-516.

30. Vermeulen T. Separation by adsorption methods. In: Drew TB, Hoopes Jr JW, eds. Advances in chemical engineering. Vol. 2. New York: Academic Press; 1958. p. 147-208.

31. Boyer PM, Hsu JT. Effects of ligand concentration on protein adsorption in dye-ligand adsorbents. Chem. Eng. Sci. 1992;47: 241-251.

32. Bhatnagar A, Kumar E, Sillanpää M. Fluoride removal from water by adsorption-A review. Chem. Eng. J. 2011;171:811-840.

33. Loganathan P, Vigneswaran S, Kandasamy J, Naidu R. Defluoridation of drinking water using adsorption processes. J. Hazard. Mater. 2013;248-249:1-19.

34. Yadav KK, Gupta N, Kumar V, Khan SA, Kumar A. A review of emerging adsorbents and current demand for defluoridation of water: Bright future in water sustainability. Environ. Int 2018;111:80-108

35. Langmuir I. The adsorption of gases on plane surfaces of glass, mica and platinum. J. Am. Chem. Soc. 1918;40:1361-1403.

36. Thomas HC. Heterogeneous ion exchange in a flowing system. J. Am. Chem. Soc. 1944;66:1664-1666.

37. Thomas HC. Chromatography: A problem in kinetics. Ann. NY. Acad. Sci. 1948;49:161-168.

38. Loebenstein WV. Batch adsorption from solution. J. Res. Natl. Bur. Stand. - A. Phys. Chem. 1962;66A:503-515.

39. Chu KH. Fixed bed sorption: Setting the record straight on the Bohart-Adams and Thomas models. J. Hazard. Mater. 2010;177:1006-1012.

40. Chu KH. Extracting surface diffusion coefficients from batch adsorption measurement data: Application of the classic Langmuir kinetics model. Environ. Technol. 2019;40:543-552.

41. Nigri EM, Cechinel MAP, Mayer DA, et al. Cow bones char as a green sorbent for fluorides removal from aqueous solutions: Batch and fixed-bed studies. Environ. Sci. Pollut. Res. 2017;24: 2364-2380.

42. Do DD. Analysis of a batch adsorber with rectangular adsorption isotherms. Ind. Eng. Chem. Fund. 1986;25:321-325.

43. Yao C, Chen T. A film-diffusion-based adsorption kinetic equation and its application. Chem. Eng. Res. Des. 2017;119:87-92.

44. Leyva-Ramos R, Rivera-Utrilla J, Medellin-Castillo NA, Sanchez-Polo M. Kinetic modeling of fluoride adsorption from aqueous solution onto bone char. Chem. Eng. J. 2010;158: 458-467.

45. Maiti A, Basu JK, De S. Chemical treated laterite as promising fluoride adsorbent for aqueous system and kinetic modeling. Desalination 2011;265:28-36.

46. Yao C, Chen T. A new simplified method for estimating film mass transfer and surface diffusion coefficients from batch adsorption kinetic data. Chem. Eng. J. 2015;265:93-99. 